\title{
Magnetic activity in the young solar analog LQ Hydrae
}

\section{Active longitudes and cycles ${ }^{\star}$}

\author{
S. V. Berdyugina ${ }^{1}$, J. Pelt $^{1,2}$, and I. Tuominen ${ }^{1}$ \\ 1 Astronomy Division, PO Box 3000, 90014 University of Oulu, Finland \\ 2 Tartu Observatory, Tõravere, 61602 Estonia
}

Received 23 May 2002 / Accepted 29 July 2002

\begin{abstract}
We present the first evidence that a single active dwarf of solar type can show a long-lived, nonaxisymmetric spot distribution - active longitudes on opposite hemispheres, similar to evolved, rapidly rotating RS CVn-type binary stars. We analyse new as well as published photometric observations of the young active dwarf LQ Hya, spanning almost 20 years. We find that activity of the star has three activity cycles: a 5.2-yr "flip-flop" cycle, a 7.7-yr period in the amplitude modulation of the brightness and an approximately 15-yr period in variations of the mean brightness. The two shorter cycles are related to the alternating active longitudes and are similar to cycles observed in RS CVn-type stars. The 15-yr cycle reflects periodic changes of the mean spottedness of the star and resembles the solar 11-year cycle. The spot rotation period (about 1.6 days) changes during the 15-yr cycle, indicating the presence of small differential rotation. The lengths of the three cycles are related as 3:2:1, with the repetition of the spot configuration after 15 years. We discuss the possibility that the observed spot cycles represent two different magnetic dynamo modes operating in LQ Hya: an axisymmetric mode, as in the Sun, and a nonaxisymmetric higher order mode with two cycles in spot patterns. Our results suggest that young stars exhibit their cycles in spot distribution, as seen in LQ Hya. This is in contrast to the conclusion based on the analysis of Ca II H\&K emission from plages. The results suggest also that the Vaughan-Preston gap represents a transition from a multiple-mode dynamo to a single-mode dynamo.
\end{abstract}

Key words. stars: activity - stars: magnetic fields - stars: starspots

\section{Introduction}

The Sun is the only star whose magnetic activity can be observed and studied in detail. It exhibits 11- and 22-year spot and magnetic cycles which have been explained by an oscillatory magnetic dynamo. In order to understand how the solar cycle could evolve since the early ages of the Sun, we investigate a sample of young single dwarfs that have just arrived on the main sequence and that, therefore, could be considered as young solar analogues.

The standard theory of solar evolution predicts that the solar luminosity increases from the zero-age main sequence (ZAMS): the zero-age Sun was a G6 star with the luminosity $L_{\text {ZAMS }}=0.7 L_{\odot}$ (cf. Stix 1989 and references therein). The solar luminosity changes throughout the main-sequence lifetime were accompanied by a loss of angular momentum and, consequently, a decrease of magnetic activity. Correlations between age and various activity signatures have been established for solar type stars (e.g. Skumanich 1972; Noyes et al. 1984; Güdel et al. 1997). Such studies derived, for instance,

Send offprint requests to: S. V. Berdyugina,

e-mail: sveta.berdyugina@oulu.fi

* Table 2 is only available in electronic form at the CDS via anonymous ftp to cdsarc.u-strasbg.fr $(130.79 .128 .5)$ or via http://cdsweb.u-strasbg.fr/cgi-bin/qcat?J/A+A/394/505 the solar zero-age rotation period $P_{\text {ZAMS }}=1.3$ and X-ray luminosity $L_{\mathrm{X}, \mathrm{ZAMS}} \sim 10^{30} \mathrm{erg} \mathrm{s}^{-1}$, corresponding to the very young and active solar-type dwarfs (Gaidos 1998). A comparison of these numbers with their current values $\left(\Omega_{\text {ZAMS }} \sim 20 \Omega_{\odot}\right.$ and $\left.L_{\mathrm{X}, \mathrm{ZAMS}} \sim 10^{3} L_{\mathrm{X}, \odot}\right)$ indicates dramatic changes, which the solar activity level has undergone during $4.6 \mathrm{Gyr}$.

It is, however, unknown how the solar cycle has evolved and at what time the present cycle properties were established. The continuous records of sunspot number go back to the beginning of the 17th century, providing a time scale of only 400 years, over which the 11- and 22-year and secular cycles have been discovered (Schwabe 1844; Hale 1913; Gleissberg 1945). The persistence of these cycles have been confirmed on longer time scales with the help of cosmogenic isotopes, as the solar activity modulates the energy spectrum of the primary galactic cosmic rays interacting with the Earth's atmosphere (cf. Eddy 1978). Traces of the 11-year cycle on a time scale of about 1400 years (590-1979 AD) have been found in ${ }^{13} \mathrm{C}$ (Cini Castagnoli et al. 2000), while the secular cycle was followed back to $30000 \mathrm{BC}$ in ${ }^{10} \mathrm{Be}$ (Beer 2001). Also, from the analysis of nuclides in lunar rocks, it is concluded that the average solar-proton fluxes over the past 100000 years are not greatly different from those observed during the last three 11-yr solar cycles (Reedy \& Marti 1991). These and similar studies allow us to trace back the solar cycles on a maximum time scale of 
several ten million years with a significant decrease of time resolution in the past (Cram 1983). The stability of the cycles over thousands of years and the total level of solar activity over millions of years indicates that the magnetic dynamo governing them has not changed significantly on these time scales. This can be understood implying that the solar luminosity and the rotational rate would change by only $5 \%$ during the last half a billion years, as seen from the studies of solar type stars. Remarkable changes of the solar dynamo are expected during the first billion years of the Sun's lifetime, as the rotational rate should have dropped by an order of magnitude during this time (from a period of 1.3 days to one of 12 days).

Since the first detection of cyclic magnetic behaviour in solar-like stars (Wilson 1978), major efforts in searching for stellar cycles have concentrated on the analysis of changes in the overall activity level, resembling the solar 11-year cycle. The activity indices commonly used are the $\mathrm{Ca}$ II $\mathrm{H} \& \mathrm{~K}$ emission, appearing due to the presence of plages (Baliunas et al. 1995), and total flux variations due to the presence of spots (e.g. Oláh et al. 2000). It was firmly established that chromospheric activity in solar-type stars declines with age, and only older stars show solar-like activity cycles (Vaughan \& Preston 1980; Vaughan 1980). Moreover, there is some kind of discontinuity in stellar chromospheric activity as a function of stellar age the Vaughan-Preston gap - separating stars with and without prominent cycles. With the data collected so far, Brandenburg et al. (1998) and Saar \& Brandenburg (1999) made an attempt to trace the time evolution of stellar magnetic cycles and, thus, the dynamo. They found that most stars with an age $t \geq 0.1 \mathrm{Gyr}$ occupy two roughly parallel branches - active and inactive. Older stars, with $t \geq 1 \mathrm{Gyr}$, can show multiple cycles on different branches. Also, very active stars (G-K-M dwarfs in binary systems) appear to occupy a third branch, superactive, and a few dwarfs and many RS CVn variables appear to delineate a transition zone between the third and the other two branches.

Recently, we found that active giants and subgiants in binary systems (RS CVn-type stars) show two persistent active longitudes, their positions separated by 180 degrees in longitude (Berdyugina \& Tuominen 1998; Berdyugina et al. 1998, 1999, 2000). At certain times, the dominant activity switches between the longitudes, showing the so-called "flip-flop" effect, with the time between consecutive switches being roughly the same. We suggested the switches to be a new tracer of stellar activity cycles seen in spot pattern changes (Berdyugina \& Tuominen 1998). Later, our conclusions for the star II Peg have been confirmed by the new analysis of extended photometric data (Rodonò et al. 2000). Our results raised the question of whether switching active longitudes exist solely in binary giants and subgiants. The only single star known so far to show a similar behaviour is FK Com (Jetsu et al. 1993; Korhonen et al. 1999, 2000, 2001), an extremely rapidly rotating giant, for which, however, the process of binary coalescence has been discussed.

Our results for RS CVn stars have been also included in the analysis by Saar \& Brandenburg (1999). These stars appeared in the active branch and also in the transition region between the active and superactive branches indicating, thus, a similar dynamo behaviour with young single stars. Therefore, one could expect that active longitudes and their alternating activity are also typical for single active stars with ages $\leq 0.1 \mathrm{Gyr}$ and, thus, for the Sun at that time.

The present paper begins the study of young single stars with ages less than 1 Gyr. The main aim of our investigation is to establish whether a cyclic behaviour is exhibited by these stars in spot patterns and overall spot activity. As was noted previously, such stars rarely display smooth cyclic variations in chromospheric plages, which become more prominent as stars become older and their average activity level decreases. We expect that younger stars with a higher level of activity exhibit their cycles in spot patterns rather than in plages. This is supported by the abovementioned similarity in dynamo behaviour of RS CVn stars and young dwarfs. Also, a detailed study of a sample of young stars will provide a "time probe" of the solar dynamo evolution.

We find LQ Hya (HD 82558) to be an attractive object for such an investigation because of its extreme activity and, also, because it can be regarded as a young solar analogue. Being at least as young as the youngest Pleiades stars, it rotates 15 times faster than the Sun $(P \approx 1 \mathrm{~d} 6)$ and has probably just arrived on the zero-age main sequence (Fekel et al. 1986). In 1982, the star was discovered to be photometrically variable and to exhibit strong optical ( $\mathrm{Ca}$ II $\mathrm{H} \& \mathrm{~K}$ and $\mathrm{H} \alpha$ ) and far-ultraviolet emissions (Eggen 1984; Fekel et al. 1986; Vilhu et al. 1991). The study of observed radial velocities shows that the star is probably single (Fekel et al. 1986). Later, a complex and strongly variable spot distribution was revealed on the stellar surface with Doppler imaging techniques (Saar et al. 1992, 1994; Strassmeier et al. 1993; Rice \& Strassmeier 1998; Donati 1999; Berdyugina et al. 2001). The fact that the observed variability of LQ Hya is caused by its magnetic activity was established by detecting widespread surface magnetic fields (Saar et al. 1992, 1994; Basri \& Marcy 1994; Donati et al. 1997; Donati 1999). Moreover, evidence for long-term evolution of a large scale magnetic field was found from Zeeman-Doppler images (Donati 1999). Kitchatinov et al. (2000) assumed the presence of a small differential rotation and derived from a dynamo model a global axisymmetric field with a cycle period of 3.2 years, which was concluded to be somewhat shorter than that suggested by Donati (1999).

The cyclic behaviour of LQ Hya has been detected from overall light variations. Jetsu (1993) reported a 6.2-yr cycle with a peak-to-peak amplitude in the $V$-band of about $0^{\mathrm{m}} 1$. Strassmeier et al. (1997) also noticed variations in the $V$-band on a time scale of 7 years. Oláh et al. (2000) reported a main period of $6.8 \mathrm{yr}$ and two low-power periods of $11.4 \mathrm{yr}$ and $2.8 \mathrm{yr}$.

In the present paper, we analyse the most comprehensive photometric observations of LQ Hya and present the first evidence that long-lived active longitudes and cycles related to them can also occur in a single dwarf star. The observations are described in Sect. 2. In Sect. 3, we perform inversions of stellar light curves and reveal active longitudes, differential rotation and cycles in spot patterns. The results obtained with the inversion technique are confirmed in Sect. 4, where the same data are analysed with the methods of time series analysis. Finally, in Sect. 5, we discuss the impact of our results on understanding the phenomenon of stellar activity in different 


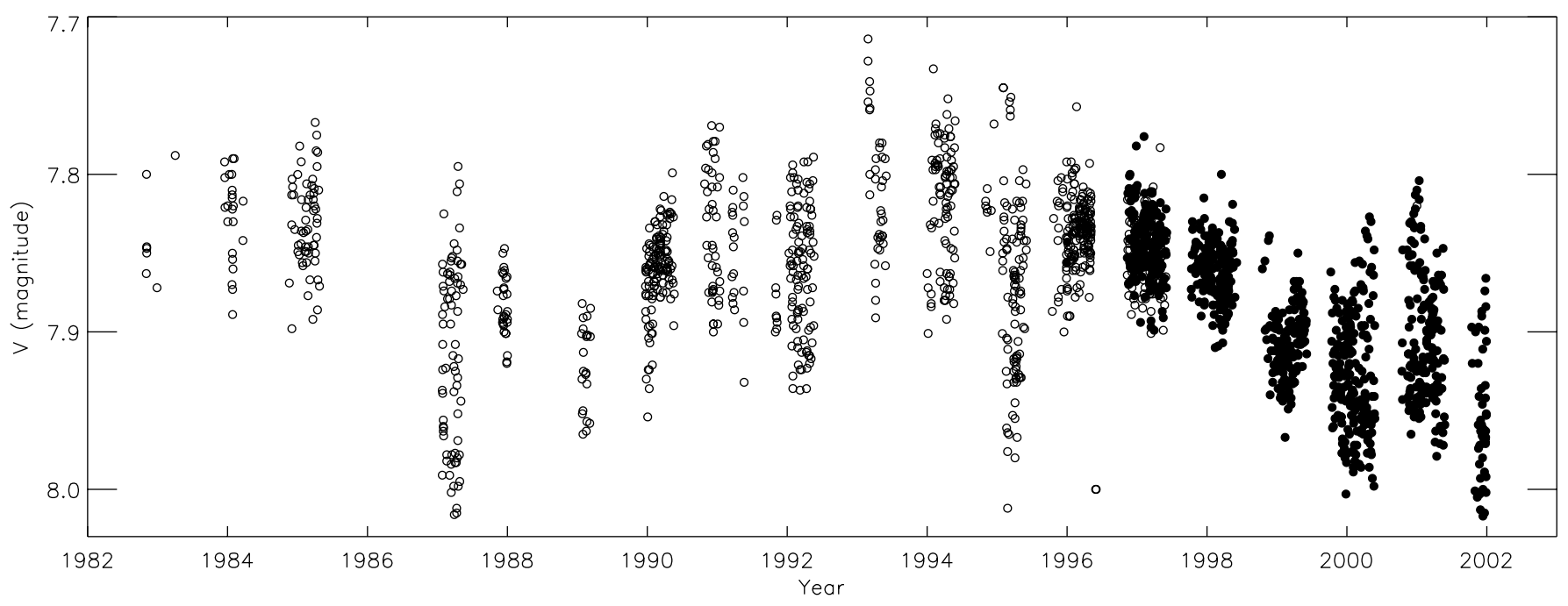

Fig. 1. The photometric data analysed in this paper. Open circles indicate previously published observations, while filled circles represent the new data.

Table 1. Sources of the light curves.

\begin{tabular}{lrl}
\hline \hline $\begin{array}{l}\text { JD range } \\
(\text { JD-2 400 000) }\end{array}$ & $N$ & Source \\
\hline $45275-45779$ & 18 & Fekel et al. (1986) \\
$45699-45734$ & 12 & Eggen (1984) \\
$46023-46178$ & 62 & Strassmeier \& Hall (1988) \\
$46023-47160$ & 155 & Boyd et al. (1990) \\
$47548-47593$ & 22 & Cutispoto (1993) \\
$47881-48760$ & 285 & Jetsu (1993) \\
$47949-47961$ & 13 & Cutispoto (1996) \\
$48331-48344$ & 13 & Cutispoto (1998a) \\
$48667-48681$ & 14 & Cutispoto (1998b) \\
$49044-50233$ & 474 & Strassmeier et al. (1997) \\
$50397-50598$ & 178 & Strassmeier et al. (1999) \\
$50078-52271$ & 946 & this paper \\
\hline
\end{tabular}

types of stars as well as the time evolution of solar and stellar dynamos.

\section{Observations}

To search for long-lived spot structures and activity cycles, we use the longest database of photometric records, of about 20 years in length, published elsewhere and newly obtained. The sources of the published observations are given in Table 1.

New photometric observations were acquired in 1995-2001 with the Phoenix 10 robotic telescope at the APT Observatory, Arizona, in the $U B V$ bands with HD 82447 as the comparison star. The errors of the observations are typically about 0.010 or less. These observations are given in Table $2^{1}$ and presented in Fig. 1.

1 Table 2 is only available in electronic form at CDS, Strasbourg, via anonymous ftp to cdsarc.u-strasbg.fr (130.79.128.5) or via

http://cdsweb.u-strasbg.fr/cgi-bin/qcat? J/A+A/394/505
For our purposes, we use only $V$-band light curves, because they represent the most complete data set. Since generally the same comparison and check stars were used in different observations, a combination of light curves from different sources was done with no corrections.

\section{Light curve inversions}

Here, we report results based on inversions of the observed light curves into stellar images - an approach that reveals the essential longitudinal spot distribution, good enough for the purpose of searching for active longitudes and their evolution (e.g. Rodonò et al. 2000).

The data were divided into 74 sets, most of them having a good phase coverage over the rotational period, but this was not the only criterion for forming a set. LQ Hya is known to show a strongly variable light curve. Therefore, the data were divided so that a given set represented a light curve stable for a given time interval. As the longest set covers an about two month period, this may indicate a time scale of light curve stability, though strong variations have been observed on shorter time scales as well. The mid-epochs of the individual sets are given in Table 3. The observations were phased with the ephemeris by Jetsu (1993): $T=2445274.22+1.601136 E$, where the zero epoch refers to one of the photometric minima.

We apply our inversion technique (Berdyugina 1998) to the photometric light curves in the two temperature approximation. The values of the photosphere and spot temperatures were adopted equal to $5250 \mathrm{~K}$ and $4400 \mathrm{~K}$, respectively. These parameters have been tested in our earlier investigation of the star (Berdyugina et al. 2001). In our calculations, we divide the stellar surface into a grid of $10^{\circ} \times 10^{\circ}$. The model assumes that, because of low spatial resolution, the intensity of each pixel on the surface, $I_{i}$, contains contributions from both temperature components, the hot photosphere, $I_{\mathrm{p}}$, and cool spots, $I_{\mathrm{s}}$, weighted by the fraction of the surface covered with spots, $f_{i}$ (spot filling factor):

$I_{i}=f_{i} I_{\mathrm{s}}+\left(1-f_{i}\right) I_{\mathrm{p}}$, 


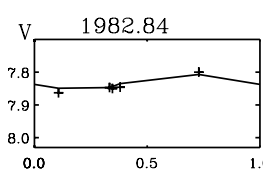

0.5
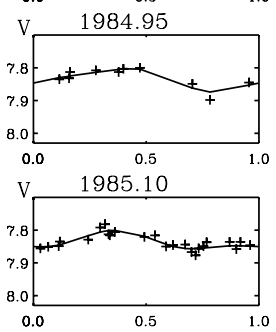

.
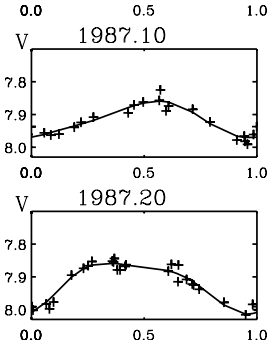

$\begin{array}{cc}0.0 & 0.5 \\ \mathrm{~V} & 1987.32\end{array}$
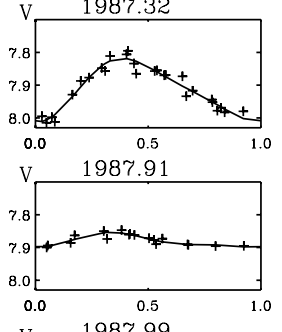

$\mathrm{V}$

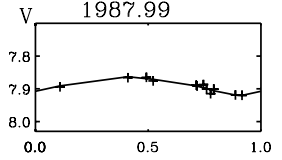

$\mathrm{V} \quad 1989.12$
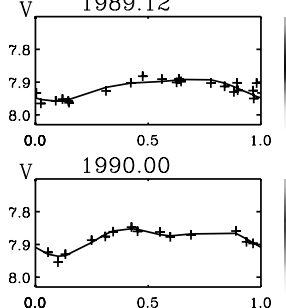
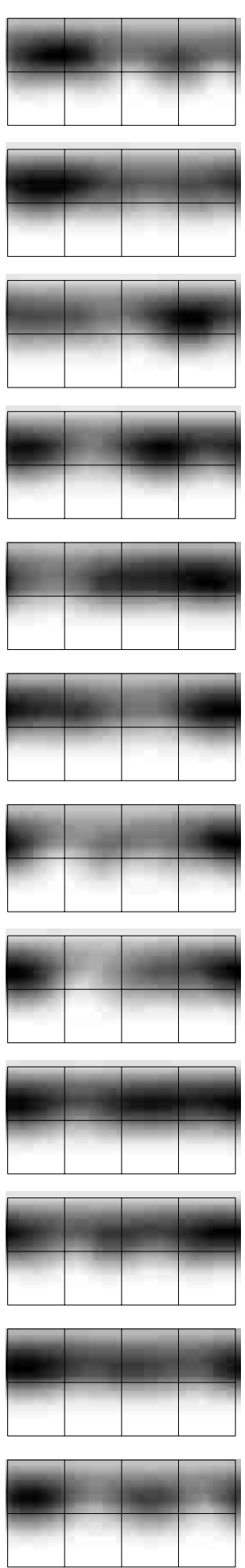
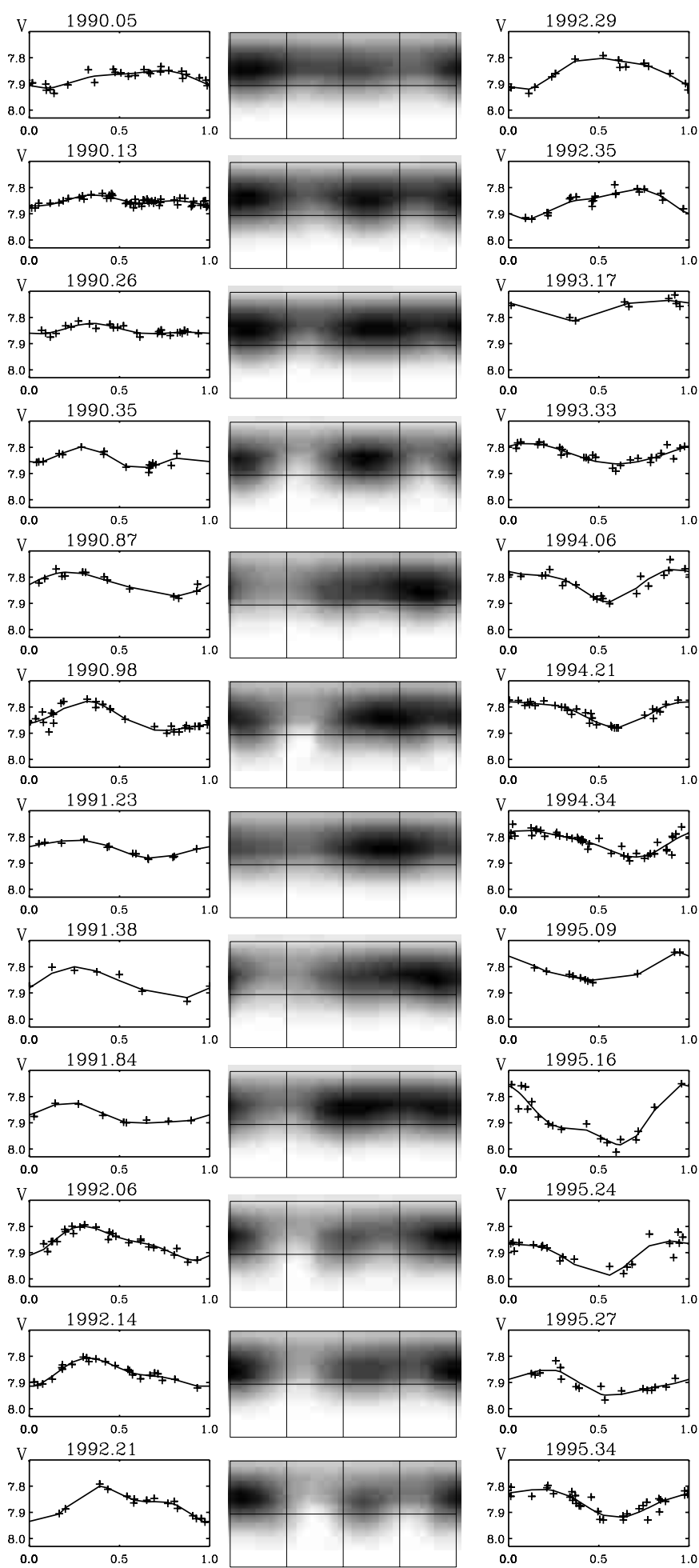
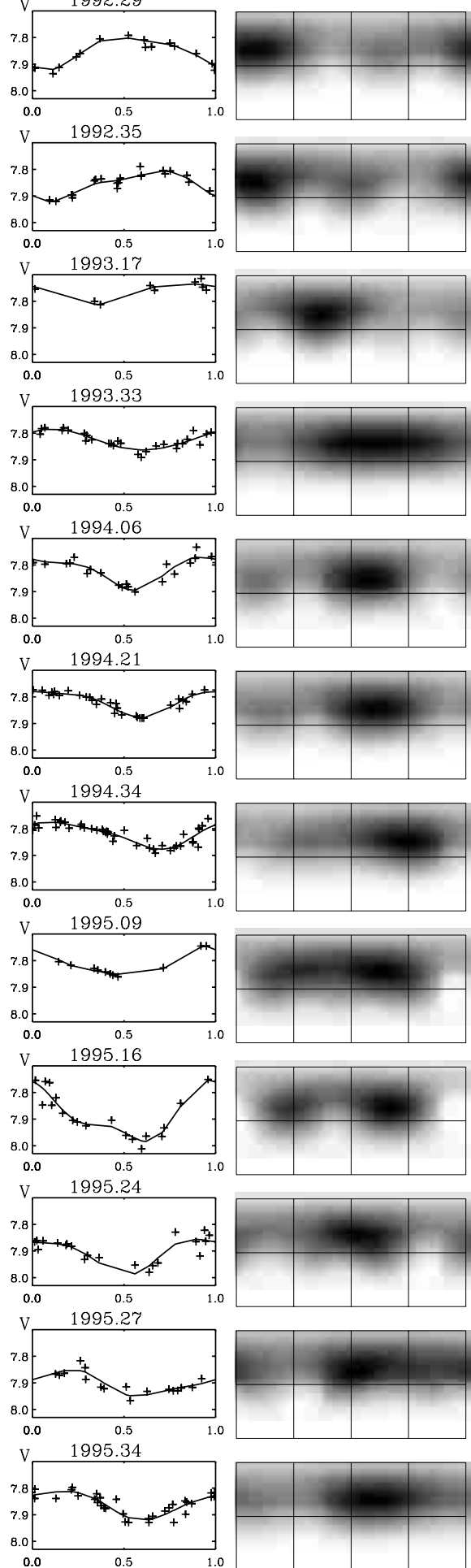

Fig. 2. The light-curve inversion results. Images are shown in a gray scale with spot filling factor being larger in darker regions. The coordinate grid in the images indicates positions of the equator and 4 longitudes separated by $90^{\circ}$. Observed and calculated $V$-band light curves are presented by crosses and lines, respectively.

with $0 \leq f_{i} \leq 1$. The instantly observed stellar flux is the integral of the intensity contributions from the surface elements seen at a given rotational phase. The inversion of the light curve results in a distribution of the spot filling factor over the stellar surface, i.e. stellar image. Figure 2 shows the light curves and the stellar images obtained from them. Since a light curve represents a one-dimensional time series, the resulting stellar image contains information on the spot distribution only in one direction, in longitudes, while spot extents and locations in latitudes remain uncertain. As a result, maximum spot concentration always appears at the central latitude of the stellar disk whose value depends on the assumed inclination of the 

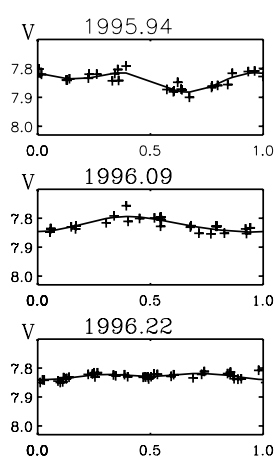

$\begin{array}{lr}0.0 & 0.5 \\ \mathrm{~V} & 1996.30\end{array}$

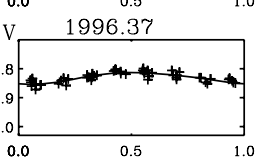

$\begin{array}{cc}0.0 & 0.5 \\ \mathrm{~V} & 1996.91\end{array}$

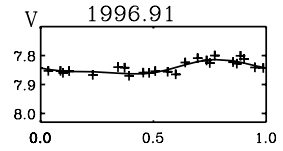

$\mathrm{V}$

\begin{tabular}{cc}
0.0 & 0.5 \\
$V$ & 1997.06 \\
\hline
\end{tabular}

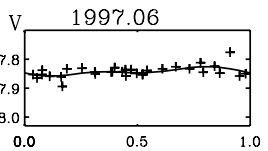

$\mathrm{V} \quad 1997.18$

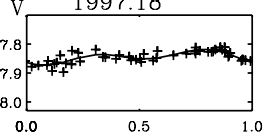

\begin{tabular}{cc}
0.0 & 0.5 \\
\hline & 1997.33
\end{tabular}

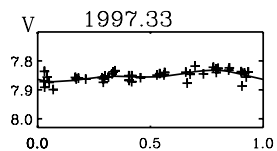

$\mathrm{V} \quad 1997.84$

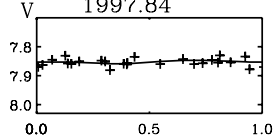

1997.96

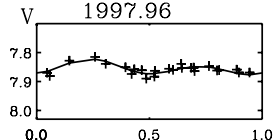

\begin{tabular}{cr}
0.0 & 0.5 \\
$\mathrm{~V}$ & 1998.04 \\
\hline
\end{tabular}
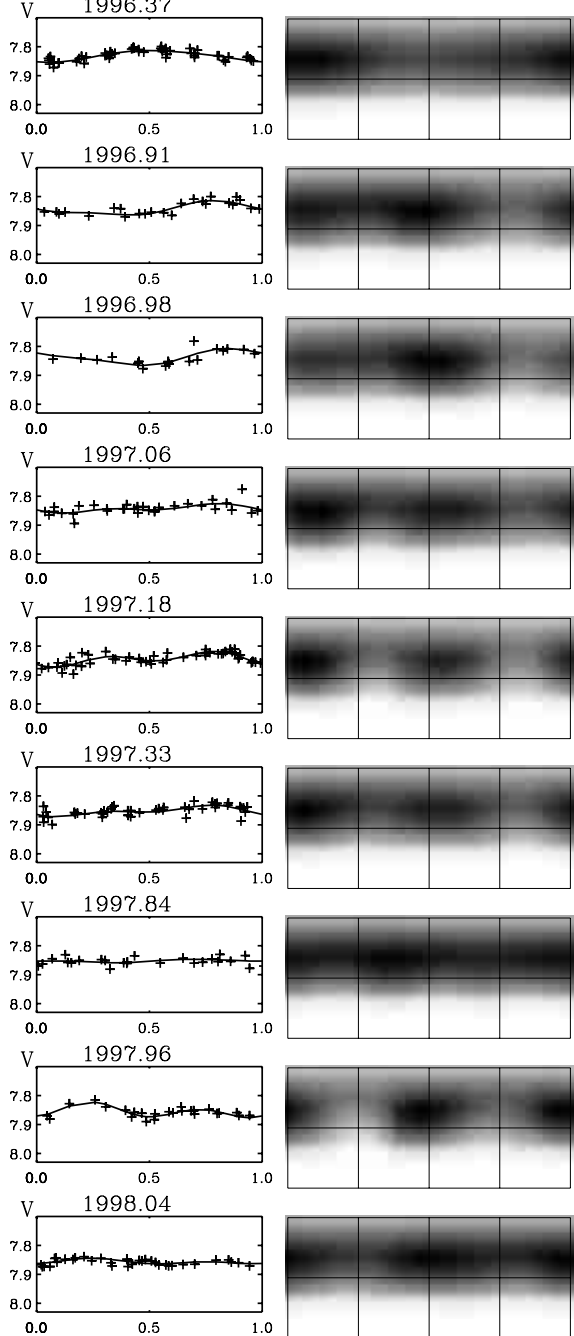

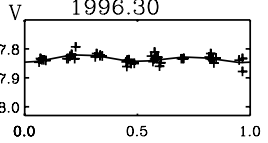

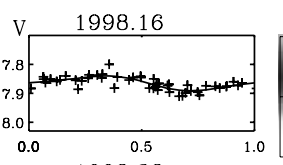
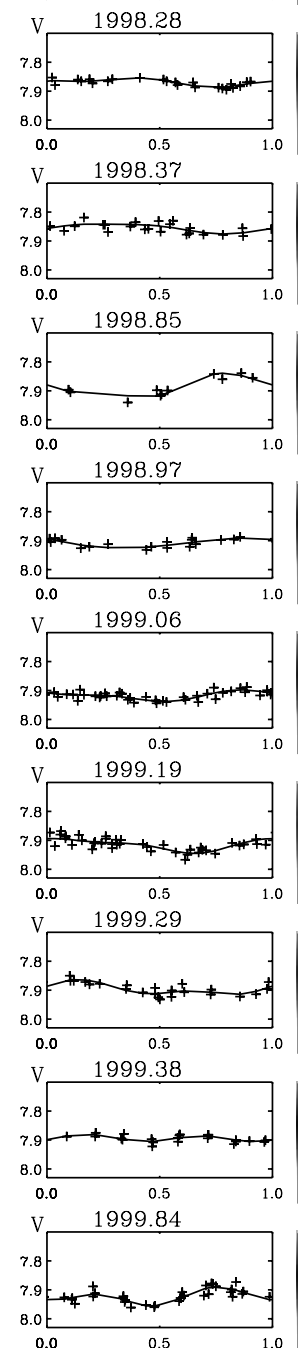

\begin{tabular}{cc}
0.0 & 0.5 \\
$\mathrm{~V}$ & 1999.95 \\
\hline
\end{tabular}
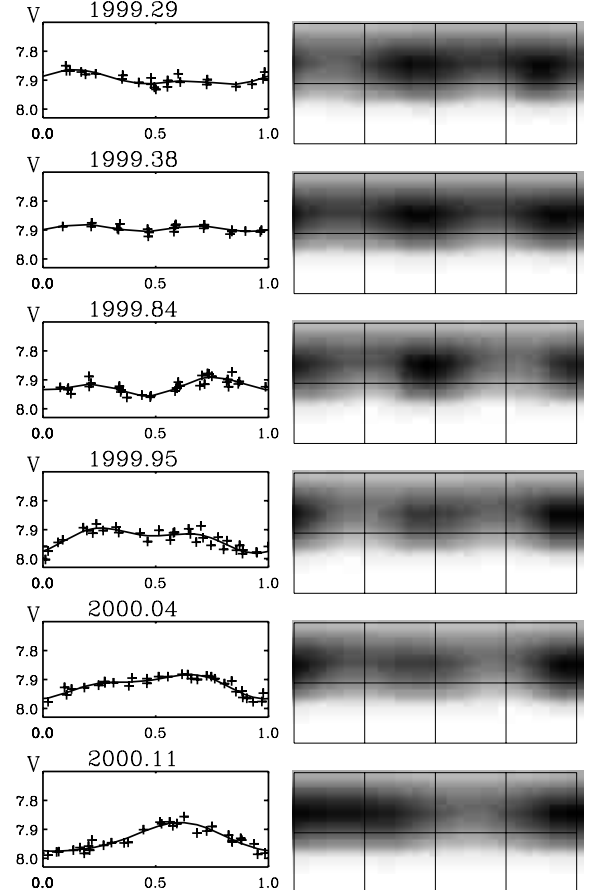
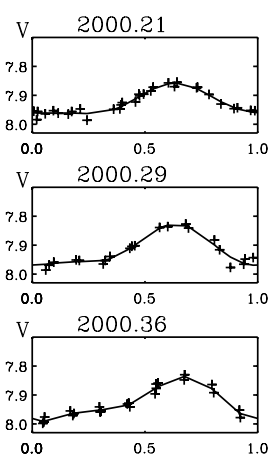

\begin{tabular}{cc}
0.0 & 0.5 \\
$\mathrm{~V}$ & 2000.85 \\
\hline
\end{tabular}
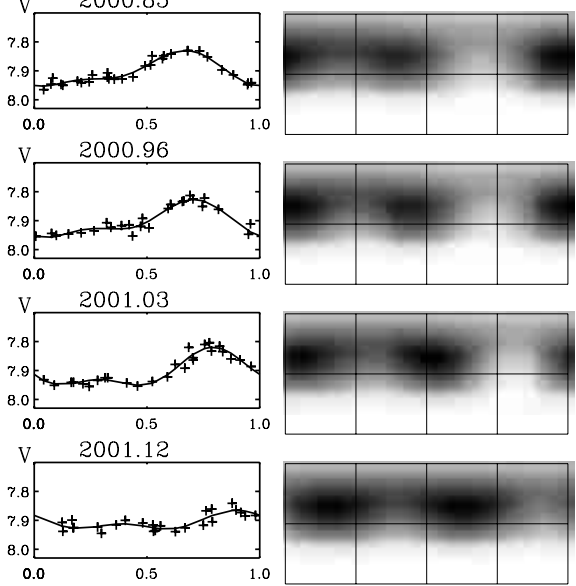

$\begin{array}{cr}0.0 & 0.5 \\ V & 2001.21\end{array}$
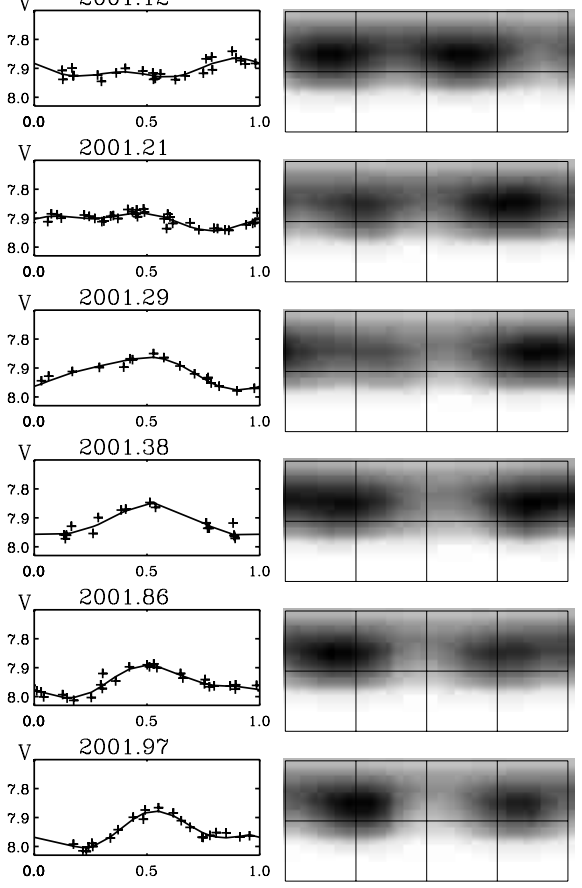

Fig. 2. continued.

rotational axis. Because of the projection effect and limb darkening, images show more well defined structures than the light curves and, therefore, are very useful for determining longitudes of spot concentrations. Although this approach is less informative than the Doppler imaging technique, based on spectroscopic observations, the analysis of more continuous and frequent photometric data allows conclusions on longitudinal spot patterns and their long-term evolution.

\subsection{Active longitudes}

From the images we recover spot longitudes at the location of the maximum spot filling factor. Two maxima are usually seen in the images, which indicate two spot concentrations on the stellar surface, with one generally being larger. The photometric phases calculated from the spot longitudes are given in Table 3. The time dependence of the spot phases is shown in Fig. 3. 
Table 3. Spot parameters: JD is the Julian date of the mid epoch of the light curve observation, $\varphi$ is the recovered phase of the active region, and $V$ is the magnitude of the star at phase $\varphi$.

\begin{tabular}{|c|c|c|c|c|c|c|c|c|c|c|c|c|c|}
\hline Set & Year & JD & $\varphi_{1}$ & $\varphi_{2}$ & $V_{1}$ & $V_{2}$ & Set & Year & JD & $\varphi_{1}$ & $\varphi_{2}$ & $V_{1}$ & $V_{2}$ \\
\hline 1 & 1982.84 & 45276 & 1.217 & 0.729 & 7.848 & 7.807 & 38 & 1996.09 & 50116 & 1.004 & - & & - \\
\hline 2 & 1984.09 & 45730 & 1.172 & - & - & - & 39 & 1996.22 & 50159 & 1.031 & 0.506 & 7.840 & 7.828 \\
\hline 3 & 1984.95 & 46046 & 0.790 & - & 7.873 & - & 40 & 1996.30 & 50191 & 0.992 & 0.500 & & 7.843 \\
\hline 4 & 1985.10 & 46102 & 0.674 & 1.071 & 7.856 & 7.850 & 41 & 1996.37 & 50217 & 1.062 & - & 7.854 & - \\
\hline 5 & 1985.26 & 46158 & 0.854 & - & 7.859 & - & 42 & 1996.91 & 50414 & 0.456 & 1.081 & 7.863 & 7.856 \\
\hline 6 & 1987.10 & 46832 & 0.938 & - & 7.969 & - & 43 & 1996.98 & 50440 & 0.506 & 1.139 & 7.863 & 7.837 \\
\hline 7 & 87.20 & 46868 & 0.968 & 0.477 & 8.013 & 7.869 & 44 & 1997.06 & 50469 & 1.105 & 0.538 & 7.858 & 7.846 \\
\hline 8 & 1987.32 & 46908 & 1.016 & - & 8.014 & - & 45 & 1997.18 & 50512 & 1.071 & 0.540 & 7.872 & 7.850 \\
\hline 9 & 1987.91 & 47126 & 1.016 & 0.631 & 7.897 & 7.888 & 46 & 1997.33 & 50568 & 1.072 & 0.528 & 7.872 & 7.855 \\
\hline 10 & 1987.99 & 47156 & 0.937 & 0.502 & 7.921 & 7.870 & 47 & 1997.84 & 50754 & 0.375 & 0.981 & 7.859 & 7.855 \\
\hline 11 & 89.12 & 47569 & 1.077 & 0.528 & 7.957 & 7.899 & 48 & 1997.96 & 50798 & 0.966 & 0.489 & 7.879 & 7.873 \\
\hline 12 & 90.00 & 47888 & 1.105 & 0.614 & 7.936 & 7.873 & 49 & 1998.04 & 50826 & 0.978 & 0.520 & 7.864 & 7.864 \\
\hline 13 & 1990.05 & 47907 & 1.081 & 0.528 & 7.915 & 7.862 & 50 & 1998.16 & 50873 & 0.698 & - & 7.891 & - \\
\hline 14 & 1990.13 & 47941 & 1.058 & 0.607 & 7.870 & 7.859 & 51 & 1998.28 & 50914 & 0.730 & 1.187 & 7.885 & 7.866 \\
\hline 15 & 90.26 & 47982 & 1.066 & 0.645 & 7.862 & 7.861 & 52 & 1998.37 & 50945 & 0.788 & - & 7.876 & - \\
\hline 16 & 90.35 & 48017 & 0.597 & 1.050 & 7.874 & 7.857 & 53 & 1998.85 & 51124 & 0.458 & 1.093 & 7.918 & 7.902 \\
\hline 17 & 1990.87 & 48209 & 0.831 & - & 7.870 & - & 54 & 1998.97 & 51165 & 0.371 & - & 7.924 & - \\
\hline 18 & 1990.98 & 48249 & 0.695 & 1.014 & 7.888 & 7.862 & 55 & 1999.06 & 51201 & 0.537 & 1.095 & 7.938 & 7.914 \\
\hline 19 & 1991.23 & 48337 & 0.678 & - & 7.880 & - & 56 & 1999.19 & 51244 & 0.661 & 1.237 & 7.945 & 7.907 \\
\hline 20 & 91.38 & 48391 & 0.867 & - & 7.917 & - & 57 & 1999.29 & 51284 & 0.851 & 0.427 & 7.914 & 7.911 \\
\hline 21 & 1991.84 & 48564 & 0.557 & 0.902 & 7.899 & 7.891 & 58 & 1999.38 & 51316 & 0.915 & 0.437 & 7.904 & 7.903 \\
\hline 22 & 1992.06 & 48644 & 0.954 & 0.537 & 7.934 & 7.850 & 59 & 1999.84 & 51482 & 0.478 & 1.007 & 7.9 & 7.938 \\
\hline 23 & 1992.14 & 48674 & 1.002 & 0.604 & 7.920 & 7.868 & 60 & 1999.95 & 51524 & 0.949 & 0.471 & 7.982 & 7.921 \\
\hline 24 & 1992.21 & 48696 & 1.054 & 0.601 & 7.981 & 7.855 & 61 & 2000.04 & 51556 & 0.977 & 0.431 & 7.967 & 7.906 \\
\hline 25 & 1992.29 & 48725 & 1.087 & 0.653 & 7.918 & 7.816 & 62 & 2000.11 & 51584 & 1.005 & - & 7.975 & - \\
\hline 26 & 1992.35 & 48748 & 1.079 & 0.497 & 7.922 & 7.837 & 63 & 2000.21 & 51617 & 0.311 & 0.955 & 7.955 & 7.955 \\
\hline 27 & 1993.17 & 49047 & 0.369 & - & 7.812 & - & 64 & 2000.29 & 51648 & 0.957 & 0.332 & 7.966 & 7.950 \\
\hline 28 & 1993.33 & 49107 & 0.590 & - & 7.863 & - & 65 & 2000.36 & 51675 & 0.997 & 0.393 & 8.001 & 7.939 \\
\hline 29 & 1994.06 & 49374 & 0.564 & 1.105 & 7.894 & 7.790 & 66 & 2000.85 & 51856 & 0.988 & 0.381 & 7.955 & 7.920 \\
\hline 30 & 1994.21 & 49427 & 0.600 & 1.158 & 7.879 & 7.788 & 67 & 2000.96 & 51895 & 1.024 & 0.430 & 7.955 & 7.925 \\
\hline 31 & 1994.34 & 49473 & 0.720 & - & 7.876 & - & 68 & 2001.03 & 51921 & 0.504 & 1.078 & 7.946 & 7.943 \\
\hline 32 & 1995.09 & 49752 & 0.615 & - & 7.838 & - & 69 & 2001.12 & 51955 & 0.613 & 0.175 & 7.928 & 7.927 \\
\hline 33 & 1995.16 & 49776 & 0.6 & 1.247 & 7.974 & 7.909 & 70 & 2001.21 & 51985 & 0.781 & 0.264 & & 7.901 \\
\hline 34 & 1995.24 & 49801 & 0.533 & 1.021 & 7.980 & 7.867 & 71 & 2001.29 & 52013 & 0.890 & - & 7.975 & - \\
\hline 35 & 1995.27 & 49814 & 0.530 & 0.955 & 7.948 & 7.899 & 72 & 2001.38 & 52040 & 0.878 & 0.179 & 7.956 & 7.949 \\
\hline 36 & 1995.34 & 49841 & 0.594 & - & 7.916 & - & 73 & 2001.86 & 52223 & 0.179 & 0.770 & 8.002 & 7.957 \\
\hline 37 & 1995.94 & 50059 & 0.670 & 1.173 & 7.882 & 7.835 & 74 & 2001.97 & 52262 & 0.240 & 0.812 & 8.004 & 7.967 \\
\hline
\end{tabular}

The two spot concentrations constitute two active longitudes about $180^{\circ}(\Delta \varphi=0.5)$ apart. As seen from Fig. 3, the active longitudes have a noticeable linear drift to earlier phases. Since the phases were calculated with a fixed period value, the rate of the drift indicates the difference between the used period value and its observed averaged value. As found from linear fits to the phases, the annual phase drift of the active longitudes is on average $-0.012 \pm 0.002$. Accounting for the drift, the correction to the rotational period appears to be as small as $(8.4 \pm 1.4) \times 10^{-5}$ day, i.e., about only $7 \mathrm{~s}$. Despite its small value, such a drift results in a quarter phase shift over 20 years, which is clearly seen in Fig. 3. Therefore, the new period value is found to be $1.601052 \pm 0.000014$.

\subsection{Differential rotation}

The new period value determined from the linear drift of the active longitudes represents, however, only the average rotation period. Actually, the rotation rate of the active longitudes is different for different years and seemingly changes periodically. This is seen from a harmonic fit to the phases shown in Fig. 3. The fit was calculated for both active longitudes simultaneously.

The period variation can be interpreted as an indication of differential rotation. Its magnitude can be estimated from the observed period variations. From the harmonic fit to the active longitudes, two extreme values of the rotation period can be found: $P_{\min }=1.600905$ and $P_{\max }=1.601198$, with the error of $4.3 \times 10^{-5}$. The difference between the periods is significant. Furthermore, according to our preliminary surface imaging results (Berdyugina et al. 2001), maximum spottedness in LQ Hya covers the latitude interval $50^{\circ}$ to $60^{\circ}$. Combining this with the $P_{\min }$ and $P_{\max }$ values and using the solar law of differential rotation in the form: $\Omega=\Omega_{0}+\Delta \Omega \sin ^{2} \psi$, we find $\Delta \Omega / \Omega_{0} \approx 0.002$, where $\Omega_{0}$ is the angular velocity at the equator and $\Delta \Omega$ is the difference between the equatorial and polar 


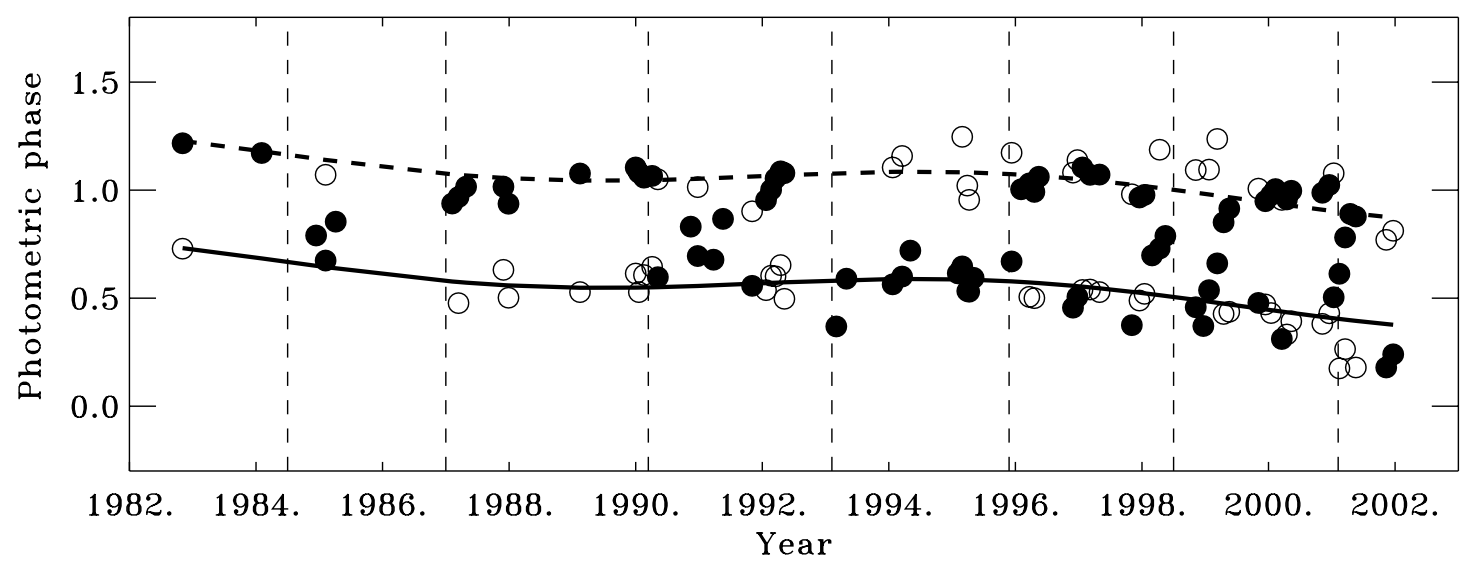

Fig. 3. Phases of the spots recovered on the surface of LQ Hya using the light curve inversion technique. Filled symbols denote larger spots. Active longitudes are traced by thick solid and dashed lines, which are harmonic fits revealing the 15-year cycle in phase variations. Vertical dashes point out the moments of the switches between the active longitudes ("flip-flops").

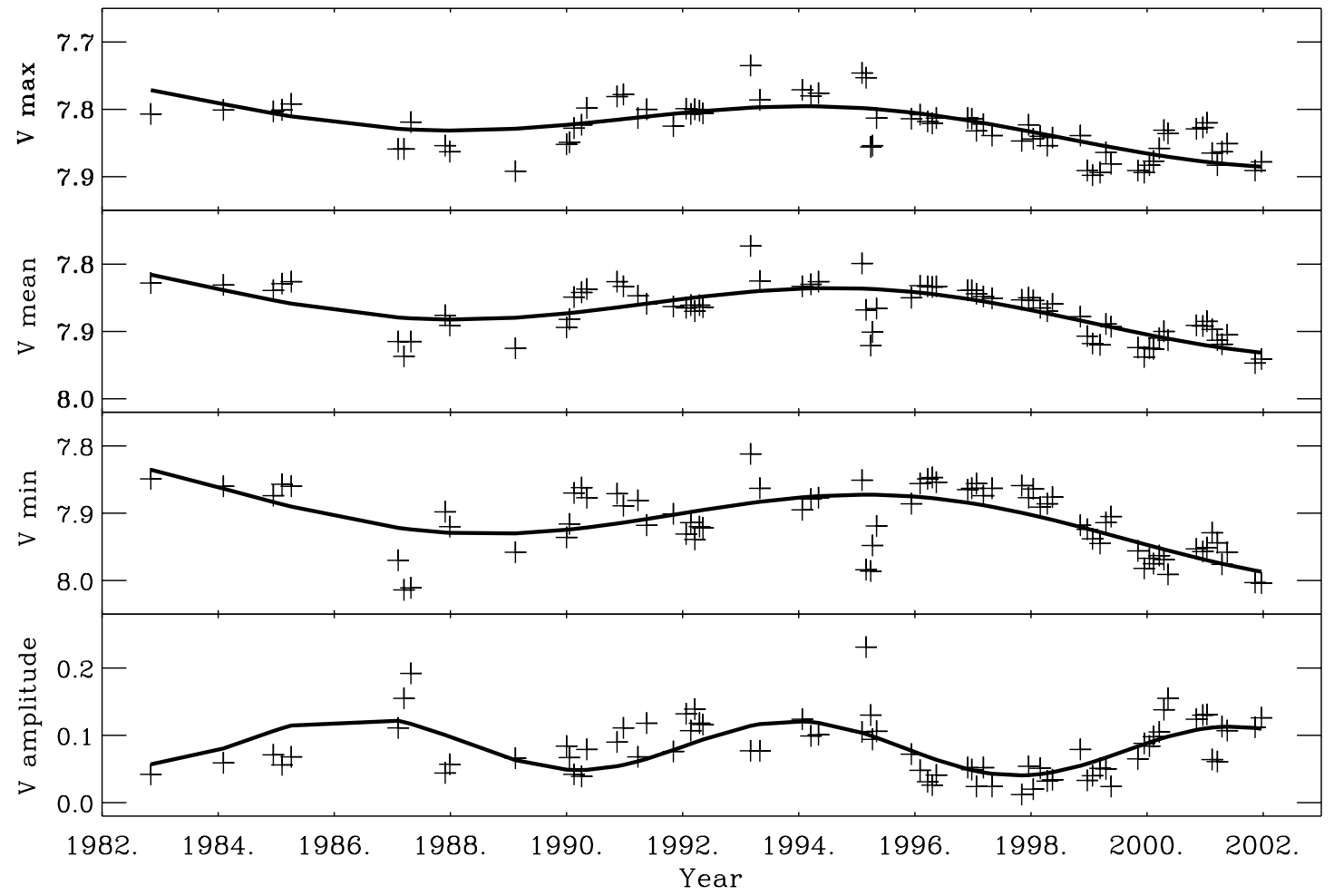

Fig. 4. $V$-band maximum, mean, minimum and peak-to-peak amplitude magnitudes measured from the individual light curves. Single harmonic fits to the variations reveal dominant cycles in the data. The maximum, mean and minimum magnitudes vary on a long-term scale of about 15 years and the amplitude clearly is modulated by the 7.7-year cycle. The minimum magnitude is also affected by the 7.7 cycle.

angular velocities. The ratio for LQ Hya is two orders of magnitude smaller than the solar value.

\subsection{Activity cycles}

A 5.2-year cycle is observed as a series of abrupt switches of the dominant activity between the active longitudes. Our results, presented in Fig. 3, show that larger spot concentrations alternately occupy the active longitudes for $2.6 \pm 0.4$ years. During the total cycle of 5.2 years, the dominant activity occupies subsequently both active longitude. Similar series of active longitude switches were observed in RS CVn stars
(Berdyugina \& Tuominen 1998) and also in FK Com and were tentatively called "flip-flops" (Jetsu et al. 1993; Korhonen et al. 2002). This name implies quick alternations in the spot distribution between two active longitudes.

A 7.7-year cycle is clearly seen in the amplitude modulation of the brightness (see the last panel of Fig. 4). It can also be traced in the behaviour of the active longitudes. As seen from Figs. 3 and 4, when the amplitude of the brightness variation is near its minimum (in the years 1990 and 1998), the larger active region apparently migrates from one active longitude to the other within about a year. A similar migration can be also noticed in the smaller active region, so that the active 

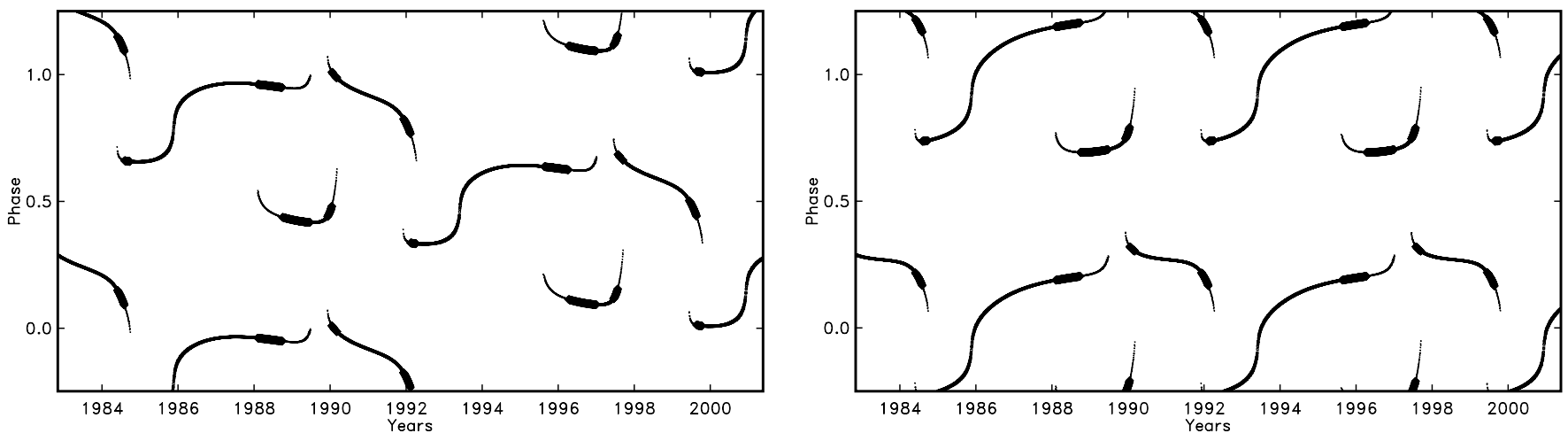

Fig. 5. Phases of the minima for the best multiperiodic approximation curve computed with the rotation periods $P=1.601136$ (left panel) and $P=1.600834$ (right panel). The main (stronger) minima are depicted with widest marks, the middle-thick marks are used to depict phases for single minima, and the narrow marks are used for secondary minima. On the right panel, the phase drift is almost gone while the cycle timing pattern is the same as on the left panel. The abrupt phase shifts after 1992 and 1999 are well seen.

longitudes apparently exchange their location. This indicates a shift of the dominant activity by $180^{\circ}$ in longitude and is reminiscent of the "flip-flop" phenomenon occurring in the 2.6-year intervals. This cycle is also confirmed by the time series analysis presented below in the Sect. 4.1.

A 15-year cycle is seen in the period variations of the active longitudes rotation (Fig. 3) and also in the maximum, minimum and mean $V$ magnitude variations (Fig. 4). The uncertainty of the cycle length is a few years, because the data set covers only about 20 years and the second global minimum of the stellar brightness is not completely covered. If, however, the zero epoch is fixed in the fitting procedure as the middle epoch between the global maximum and minimum (inflection point), namely about the year 1991, the uncertainty of the cycle length is reduced to half a year. Further observations of the star will certainly improve our estimate. A correlation of the period variation with the mean brightness of the star during the 15-year cycle is reminiscent of the solar 11-year cycle, i.e., spots in the course of the cycle seem to arise at different latitudes and rotate with different periods because of differential rotation. A kind of butterfly diagram is expected therefore for LQ Hya. It can be obtained with the help of the surface imaging technique. This is the aim of our second paper of this series.

Note that the lengths of the cycles appear to be in a certain relation: $5.2 \times 3 \approx 7.7 \times 2 \approx 15$ years. Such a relation can be understood if one considers the interference of the cycles. The 5.2-yr cycle is seen in the active longitude alternation every 2.6 years. The $7.7-y r$ cycle interfere with the 5.2 -yr cycle as follows: during the first 5.2 years the dominant activity occupies subsequently both active longitudes and, for the rest of the cycle, after a regular switch of the activity, the larger active region migrates from one active longitude to the other and stays there until the next switch. Such migrations occur therefore in every third 2.6-yr interval. The configuration of the active longitudes is repeated over the 15 -yr cycle.

Finally, we conclude that the activity of LQ Hya is multicyclic and governed by $(i)$ long-term total spot area changes on a time scale of about 15 years and (ii) two interfering mechanisms of spot area redistribution between the active longitudes on the time scales of 5.2 and 7.7 years.

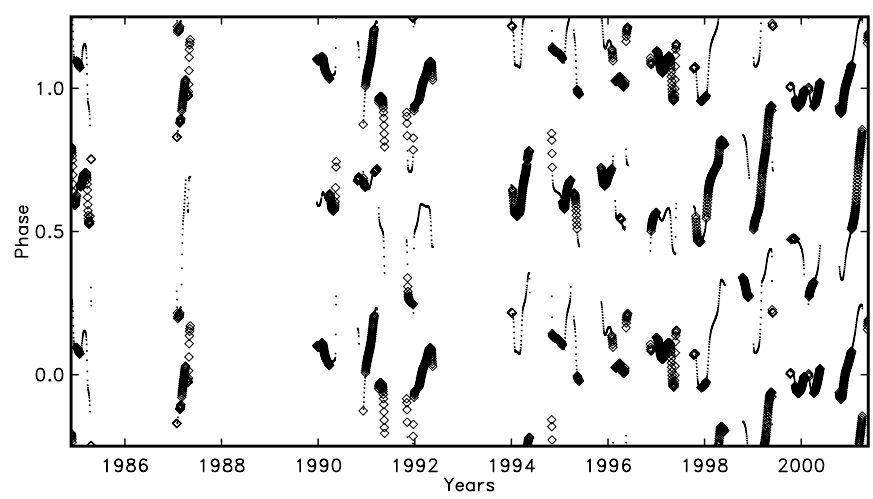

Fig. 6. Phases of minima for the carrier fit approximation computed with the base period $P=1^{\mathrm{d}} .601136$. The main minima are depicted with diamonds and secondary minima with points. The overall pattern is somewhat similar to the inversion analysis results. The longer time gaps in the plot are due to data sparsity in some seasons. The carrier fit method needs more data points to produce safe approximations.

\section{Time series analysis}

To check the validity of the above results we analysed the same data set with two different time series analysis methods. First, we searched for a global multiperiodic approximation to the data and, second, we used the so-called "carrier fit" method to analyse the local behaviour of the light curve. In this analysis we do not make any physical assumptions.

\subsection{Global approximation}

In our first model we assume that the full data set can be described with one basic period which is somehow periodically modulated. In this way we seek effectively only two generic periods $P_{1}=1 / v_{1}$ and $P_{2}=1 / v_{2}$ to describe the regular part of the variability. The most general model for such a waveform will contain all positive frequencies from the family

$v_{i, j}=i \times v_{1}+j \times v_{2}, i, j=-N, \ldots, N$,

where $N$ is a certain cutoff to the full (infinite) model. In our analysis, we choose $N=2$ as an appropriate value to avoid 
overfit and lengthy computations. To determine the best values of the generic periods we computed least square fits (with all coupling frequencies included) for a full set of trial pairs $P_{1}=1.58 \ldots 1.63, P_{2}=1000 \mathrm{~d} \ldots 3000$. The best pair occurred to be $P_{1}=1$ d $600834, P_{2}=2748.11=7.52$ years. The corresponding fit to the observations is not very good (the reduction of the sum of squares of residuals $R^{2}=31.6 \%$ ) but the result is nevertheless quite interesting.

In Fig. 5 (left panel), the phases of the minima of the best fitting model computed with the rotation period $P=1^{\mathrm{d}} .601136$ determined by Jetsu (1993) are displayed. This period appeared to be too long, as was also found in Sect. 3, since there is a clear drift of the phase to smaller values. If the minima are computed using the base period $P=1_{1}^{\mathrm{d}} 60083$ (Fig. 5, right panel), the phase drift is practically gone and the full variability is periodic with the modulation period. One can see that the overall structure of the activity is similar to the pattern we obtained with the inversion technique. The base period coincides within the error with $P_{\min }$ as determined in Sect. 3. The best modulation period of 7.52 years is seen as abrupt phase shifts in 1992 and 1999. It corresponds to the 7.7-year cycle discussed above.

\subsection{Carrier fit method}

While the modulation analysis of the previous subsection assumed a global persistent periodicity, the carrier fit method is based on the assumption that the period, its phase, and amplitude can all change with time. A full introduction to this relatively new method will be given elsewhere. Here we only mention that it is based on the local approximation of data fragments by the following model:

$f(t)=a(t) \cos \left(2 \pi v_{0} t\right)+b(t) \sin \left(2 \pi v_{0} t\right)$,

where $a(t)$ and $b(t)$ are slowly changing amplitudes which are modelled by using splines, and $v_{0}$ is the so-called carrier frequency. An approximation of this kind can be used to model narrow band stochastic curves as well as curves with mildly changing phases and amplitudes.

In Fig. 6, the result of the carrier fit analysis is displayed. All patterns of the minima are similar to results of the inversion analysis presented in Sect. 3. This is not of course unexpected because the spot mapping by inversion can also be regarded as a procedure to approximate smoothly observed light curves.

\section{Discussion}

Although detailed theoretical models are needed to understand the behaviour of the activity of LQ Hya, we can preliminarily address some questions.

\subsection{Is LQ Hya a good young solar analogue?}

The complicated behaviour of the spot activity of LQ Hya occurring on various time scales raises the questions whether this star can truly represent the young Sun and, if so, at what age. Young solar-mass stars are remarkable by their high rotation rate, chromospheric activity, spottedness, and coronal $\mathrm{X}$-ray emission. Also, lithium abundance and space motions are good indicators of stellar age. Basically, three observables give major evidence for the early age of LQ Hya: rapid rotation, $P=1.6$ days, high X-ray luminosity, $L_{\mathrm{X}} / L=0.45 \times 10^{-3}$, and lithium overabundance, $\log \epsilon(\mathrm{Li})=2.8$ (Hünsch et al. 1999; Fekel et al. 1986). Also, the galactic velocity components indicate clearly that LQ Hya is a young-disk star and certainly younger than 0.3 Gyr (Jeffries 1995). Comparing LQ Hya's observables with those the Sun is supposed to have at various ages (Skumanich 1972; Stix 1989; Pinsonneault et al. 1989; Gaidos 1998), we find that LQ Hya corresponds to the Sun at the age of about $60 \mathrm{Myr}$, which is in accordance with the conclusion by Fekel et al. (1986).

\subsection{Do binary and single stars have similar dynamo behaviour?}

The evolution of the angular momentum in single and binary stars is known to be different: while the former spin down with time, the latter become tidally locked at fast rotation by a close companion. Therefore, for a given spectral class, the distribution of stellar rotation periods shows a wider range for binaries. Since the rotation plays a key role in stellar magnetic activity, a wider range of activity in binary stars is also observed (see e.g. Saar \& Brandenburg 1999, who found binaries in all activity branches). On the other hand, some similarities between evolved binary components and young stars, rotating with similar rates, can be expected, if the binarity has little effect on the dynamo. Therefore, the long-lived active longitude structure observed in evolved components of binary systems, such as RS CVn-type stars (Berdyugina \& Tuominen 1998), can be searched in single stars with similar rotational rates.

Nonlinear magnetic dynamo calculations show that indeed active longitudes can be dominant in both single and binary stars (Moss et al. 1995; Moss \& Tuominen 1997; Tuominen et al. 1999). Our present results for LQ Hya provide the first observational confirmation of these calculations for single stars. This preliminary result suggests that indeed the dynamo behaviour is strongly determined by the rotational rate rather than the binarity, though further investigations of single active stars are needed.

\subsection{How does the stellar dynamo evolve?}

The geometry and behaviour of stellar magnetic fields are globally determined by the stability of dynamo modes with different symmetry (e.g. Brandenburg et al. 1989). For instance, basic features of the present solar dynamo can be explained by axisymmetric mean-field dynamo models which are antisymmetric with respect to the equator (type $\mathcal{A} 0$ ). In more active stars, rotating more rapidly, a higher order nonaxisymmetric mode is excited and stable, either symmetric with respect to the equatorial plane $\mathcal{S} 1$ (Moss et al. 1995) or antisymmetric with respect to the equatorial plane $\mathcal{A} 1$ (Tuominen et al. 1999). The magnetic field configuration in such modes consists of two magnetic spots of opposite polarities - active longitudes $180^{\circ}$ apart. Beside the symmetry of the modes, their oscillatory properties are also different: while axisymmetric modes show clear cyclic behaviour (as in the solar cycle), the present models of nonaxisymmetric modes appear without oscillations. 
The switching active longitudes observed in LQ Hya and RS CVn stars favour, however, periodically oscillating nonaxisymmetric fields, which have not yet been encountered in the theoretical models. Moreover, in LQ Hya, the mechanisms governing its activity described in Sect. 3.3 suggest a dual dynamo action: the long-term total spot area changes indicate an oscillating axisymmetric mode (say $\mathcal{A} 0$ like in the Sun) with a $15-\mathrm{yr}$ period, and the active longitudes reveal the presence of a nonaxisymmetric mode (either $\mathcal{A} 1$ or $\mathcal{S} 1$ ) with two periods of 5.2 and 7.7 years. Perhaps the coexistence of the axisymmetric and nonaxisymmetric modes results in the appearance of oscillating nonaxisymmetric fields, i.e. alternating active longitudes. The relative strengths of the two dynamo modes and the period of the oscillations of the axisymmetric mode should then define the amplitudes and the lengths of the observed cycles. In LQ Hya, the ratio of the cycle lengths was determined as $1: 2: 3$, which resembles a harmonic sequence in the periods of the excited dynamo modes. We note, however, that for determining the symmetry of the dynamo modes, for both solar-type and RS CVn stars, observations of magnetic polarity of the two active longitudes are needed.

As was mentioned, $\mathcal{A} 0$ is the principle dynamo mode governing the solar 22-yr cycle. Since LQ Hya can represent the Sun in the very beginning of its main sequence evolution, our results suggest that, due to extremely rapid rotation, both axi- and nonaxisymmetric modes of comparable strengths were excited in the young Sun. As the Sun evolved and spun down, the nonaxisymmetric mode was quenched. In the present Sun, only weak traces of this mode were observed as shortlived active longitudes (e.g. Bumba \& Howard 1965; Vitinskij 1969; Mikhailutsa \& Makarova 1994) or preferred hemispheres (Balthasar \& Schüssler 1984).

The change of the dominant dynamo mode from strongly nonaxisymmetric to strongly axisymmetric as the star evolves can be responsible for the appearance of the so-called VaughanPreston gap (Vaughan \& Preston 1980; Vaughan 1980). The difference in the configuration of the magnetic field and the total energy output in these modes can result in a discontinuity in the activity level with stellar age. A similar idea was discussed by Durney et al. (1981), who suggested that strong and random variations in chromospheric emission (no cyclic variations) in young stars can occur due to multiple excited dynamo modes.

As LQ Hya and the Sun represent stars on different sides of the Vaughan-Preston gap, our results suggest that (i) young stars do exhibit their cycles, but in spot patterns, (ii) the activity of young and old stars is governed by different dynamo modes, and (iii) the Vaughan-Preston gap indeed represents a transition from a mutiple-mode dynamo to a single-mode dynamo. With a sample of single stars of different ages, we are going to test these suggestions in future investigations.

\section{Conclusions}

We present new photometric data of the single young dwarf of solar type, LQ Hya, and analyse the most comprehensive series of observations with the technique of inversion of light curves into stellar images. Such an approach reveals essentially the longitudinal spot distribution and is good enough for the purpose of studying active longitudes and their evolution. The following new results are obtained:

1. Two active longitudes about $180^{\circ}$ apart are found to dominate the activity of LQ Hya over 20 years, which resembles the activity of evolved stars in binary systems rotating with similar rates (RS CVn stars). Thus, this is the first observational evidence that long-lived, nonaxisymmetric magnetic fields can be generated in a single, rapidly rotating dwarf.

2. A new average period of the spot rotation of $1.601052 \pm$ 0.000014 days has been determined from the long-term drift of the active longitudes.

3. Surface differential rotation has been inferred from the cyclic changes of the phases of the active longitudes. Its magnitude was estimated to be as small as $\Delta \Omega / \Omega_{0} \approx 0.002$.

4. Three activity cycles have been detected in spot patterns: the 15-year cycle of the total spottedness variation coupled with the differential rotation, the 7.7-year cycle of the amplitude modulation of the brightness accompanied by the apparent migration of the larger active region from one active longitude to the other, and the 5.2-year cycle of the alternating active longitude domination. The lengths of the cycles are related as follows: $5.2 \times 3 \approx 7.7 \times 2 \approx 15$ years.

5. According to the theoretical models, the shorter cycles, occurring due to the active longitude evolution, can be identified with a nonaxisymmetric dynamo mode, while the longest cycle appears to be due to an oscillating axisymmetric mode like in the present Sun. Since LQ Hya can represent the Sun at the age of about $60 \mathrm{Myr}$, the activity of the young, rapidly rotating Sun could consist of two dynamo modes, the zero order $\mathcal{A} 0$ and a higher order mode, say $\mathcal{A} 1$. During solar evolution, as the Sun was losing its angular momentum, the power of the higher order mode should have significantly decreased, and only weak traces of it can be found in the present Sun.

6. Since LQ Hya and the Sun are good representatives of stars on different sides of the Vaughan-Preston gap, our results give the first observational evidence that the gap represents a transition from a mutiple-mode dynamo to a single-mode dynamo. Moreover, in contrast to the opinion that young stars do not show smooth cyclic activity, based on the analysis of emission from plages, our results suggest that these stars do exhibit cycles, but in spot patterns, as is seen in LQ Hya.

7. The similar activity pattern of LQ Hya and RS CVn stars - alternating active longitudes - suggests that binarity has no strong effect on the dynamo, and it is the rapid rotation that determines the dynamo behaviour of stars.

Acknowledgements. We thank Dr. G. Cutispoto for providing his observations and also Drs. M. J. Korpi, G. Rüdiger, M. Schüssler, S. Solanki, I. Usoskin for very useful discussions. JP was partially supported by Grant No. 4697 of the Estonian Science Foundation.

\section{References}

Baliunas, S. L., Donahue, R. A., Soon, W. H., et al. 1995, ApJ, 438, 269

Balthasar, H., \& Schüssler, M. 1984, Sol. Phys., 93, 177

Basri, G., \& Marcy, G. W. 1994, ApJ, 431, 844

Beer, J. 2001, Space Sci. Rev., 94, 53

Berdyugina, S. V. 1998, A\&A, 338, 97 
Berdyugina, S. V., \& Tuominen, I. 1998, A\&A, 336, L25

Berdyugina, S. V., Berdyugin, A. V., Ilyin, I., \& Tuominen, I. 1998, A\&A, 340, 437

Berdyugina, S. V., Berdyugin, A. V., Ilyin, I., \& Tuominen, I. 1999, A\&A, 350, 626

Berdyugina, S. V., Berdyugin, A. V., Ilyin, I., \& Tuominen, I. 2000, A\&A, 360, 272

Berdyugina, S. V., Ilyin, I., \& Tuominen, I. 2001, in The Eleventh Cambridge Workshop on Cool Stars, Stellar Systems, and the Sun, ed. R. J. García López, R. Rebolo, \& M. R. Zapatero Osorio (ASP, San Francisco), ASP Conf. Ser., 223, CD-1207

Brandenburg, A., Krause, F., Meinel, R., Moss, D., \& Tuominen, I. 1989, A\&A, 213, 411

Brandenburg, A., Saar, S. H., \& Turpin, C. R. 1998, ApJ, 498, L51

Boyd, L. J., Genet, R. M., Hall, D. S., Busby, M. R., \& Henry, G. W. 1990, IAPPP Commun., 42, 44

Bumba, V., \& Howard, R. 1965, ApJ, 141, 1492

Cini Castagnoli, G., Bonino, G., Taricco, C., \& Bernasconi, S. M. 2000, in Proc. of 1st Solar \& Space Weather Euroconference, The Solar Cycle and Terrestrial Climate, ed. A. Wilson, ESA SP-463, 481

Cram, L. E. 1983, in Solar and Stellar Magnetic Fields: Origins and Coronal Effects, ed. J. O. Stenflo, IAU Symp., 102, 515

Cutispoto, G. 1993, A\&AS, 102, 655

Cutispoto, G. 1996, A\&AS, 119, 281

Cutispoto, G. 1998a, A\&AS, 127, 207

Cutispoto, G. 1998b, A\&AS, 131, 321

Donati, J.-F., Semel, M., Carter, B. D., Rees, D. E., \& Cameron, A. C. 1997, MNRAS, 291, 658

Donati, J.-F. 1999, MNRAS, 302, 457

Durney, B. R., Mihalas, D., \& Robinson, R. D. 1981, PASP, 93, 537

Eddy, J. A. 1978, in The New Solar Physics, ed. J. A. Eddy, AAAS Selected Symp., 17, 11

Eggen, O. J. 1984, AJ, 89, 1358

Fekel, F. C., Bopp, B. W., Africano, J. L., et al. 1986, AJ, 92, 1150

Gaidos, E. J. 1998, PASP, 110, 1259

Gleissberg, W. 1945, Obs., 66, 123

Güdel, M., Guinan, E. F., \& Skinner, S. L. 1997, ApJ, 483947

Hale, G. E. 1913, ApJ, 38, 27

Hünsch, M., Schmitt, J. H. M. M., Sterzik, M. F., \& Voges, W. 1999, A\&AS, 135, 319

Jeffries, R. D. 1995, MNRAS, 273, 559

Jetsu, L. 1993, A\&A, 276, 345

Jetsu, L., Pelt, J., \& Tuominen, I. 1993, A\&A, 278, 449

Kitchatinov, L. L., Jardine, M., \& Donati, J.-F. 2000, MNRAS, 318, 1171

Korhonen, H., Berdyugina, S. V., Hackman, T., et al. 1999, A\&A, 346, 101
Korhonen, H., Berdyugina, S. V., Hackman, T., Strassmeier, K. G., \& Tuominen, I. 2000, A\&A, 360, 1067

Korhonen, H., Berdyugina, S. V., Strassmeier, K. G., \& Tuominen, I. 2001, A\&A, 379, L30

Korhonen, H., Berdyugina, S. V., \& Tuominen, I. 2002, A\&A, 390, 179

Mikhailutsa, V. P., \& Makarova, V. V. 1994, Sol. Phys., 155, 391

Moss, D., Barker, D. M., Brandenburg, A., \& Tuominen, I. 1995, A\&A, 294, 155

Moss, D., \& Tuominen, I. 1997, A\&A, 321, 151

Noyes, R. W., Hartmann, L. W., Baliunas, S. L., Duncan, D. K., \& Vaughan, A. H. 1984, ApJ, 279, 763

Oláh, K., Kolláth, Z., \& Strassmeier, K. G. 2000, A\&A, 356, 643

Pinsonneault, M. H., Kawaler, S. D., Sofia, S., \& Demarque, P. 1989, ApJ, 338, 424

Reedy, R. C., \& Marti, K. 1991, in The Sun in Time, A92-46664 19-92 (Tucson, University of Arizona Press) 260

Rice, J. B., \& Strassmeier, K. G. 1998, A\&A, 336, 972

Rodonò, M., Messina, S., Lanza, A. F., Cutispoto, G., \& Teriaca, C. 2000, A\&A, 358, 624

Saar, S. H., \& Brandenburg, A. 1999, ApJ, 524, 295

Saar, S. H., Piskunov, N. E., \& Tuominen, I. 1992, in The Seventh Cambridge Workshop on Cool Stars, Stellar Systems, and the Sun, ed. M. S. Giampapa, \& J. A. Bookbinder (ASP, San Francisco), ASP Conf. Ser., 26, 259

Saar, S. H., Piskunov, N. E., \& Tuominen, I. 1994, in The Eighth Cambridge Workshop on Cool Stars, Stellar Systems, and the Sun, ed. J. P. Caillault (ASP, San Francisco), ASP Conf. Ser., 64, 661

Schwabe, H. 1844, Astron. Nachr., 21, 233

Skumanich, A. 1972, ApJ, 171, 565

Stix, M. 1989, The Sun: An Introduction (Springer-Verlag, Berlin)

Strassmeier, K. G., \& Hall, D. S. 1988, ApJS, 67, 453

Strassmeier, K. G., Rice, J. B., Wehlau, W. H., Hill, G. M., \& Matthews, J. M. 1993, A\&A, 268, 671

Strassmeier, K. G., Bartus, J., Cutispoto, G., \& Rodonó, M. 1997, A\&AS, 125, 11

Strassmeier, K. G., Serkowitsch, E., \& Granzer, Th. 1999, A\&AS, 140, 29

Tuominen, I., Berdyugina, S. V., Korpi, M. J., \& Rönty, T. 1999, in Stellar Dynamos: Nonlinearity and Chaotic Flows, ed. M. Núnez, \& A. Ferriz Mas (ASP, San Francisco), ASP Conf. Ser., 178, 195

Tuominen, I., Berdyugina, S. V., Korpi, M. J. 2002, AN, 323, 367

Vaughan, A. H., \& Preston, G. W. 1980, PASP, 92, 385

Vaughan, A. H. 1980, PASP, 92, 392

Vilhu, O., Gustafsson, B., \& Walter, F. M. 1991, A\&A, 241, 167

Vitinskij, I. J. 1969, Sol. Phys., 7, 210

Wilson, O. C. 1978, ApJ, 226, 379 